\title{
Brassicaceae endémicas del Perú
}

\section{Christhian Monsalve ${ }^{1}$}

Museo de Historia Natural, Av. Arenales 1256, Aptdo. 14-0434, Lima 14, Perú christhianml@hotmail.com

\section{Resumen}

La familia Brassicaceae es reconocida en el Perú por presentar 27 géneros y 111 especies (Brako \& Zarucchi, 1993; Ulloa Ulloa et al., 2004), principalmente hierbas. En este trabajo reconocemos 34 endemismos en 12 géneros. Dos géneros, Catadysia y Dactylocardamum, son también endémicos del Perú. Estos taxones endémicos se encuentran principalmente en las regiones Puna Húmeda y Seca y Altoandina, creciendo por encima de los $3000 \mathrm{~m}$ de altitud. Se aplicaron las categorías y criterios de la UICN a 31 taxones. Seis especies endémicas han sido registradas en el Sistema Nacional de Áreas Naturales Protegidas por el Estado.

Palabras claves: Brassicaceae, Catadysia, Dactylocardamum, Perú, endemismo, plantas endémicas.

\section{Abstract}

The Brasicaceae are represented in Peru by 27 genera and 111 species (Brako \& Zarucchi, 1993; Ulloa Ulloa et al., 2004), mostly herbs. Here we recognize 34 endemic taxa in 12 genera. Two genera, Catadysia and Dactylocardamum, are also endemic to Peru. These endemic taxa are found mainly in the Very Humid and Dry Puna, and High-Andean regions, growing above $3000 \mathrm{~m}$ elevation. We applied IUCN categories and criteria to 31 endemic taxa. Six endemic species are known from the Peruvian park system.

Keywords: Brasicaceae, Catadysia, Dactylocardamum, Peru, endemism, endemic plants.

\section{Aschersoniodoxa pilosa Al-Shehbaz}

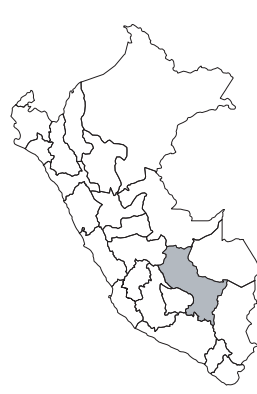
10. 1990. Herbarios: MO.

SINANPE: SHMP

\section{EN, B1a}

Publicación: Syst. Bot. 15(3): 390, f. 2, 9,

Colección tipo: B. Peyton \& T. Peyton 112

Nombre común: Ckorihuackacc.

Registro departamental: CU.

Regiones Ecológicas: AA; $4500 \mathrm{~m}$.

Herbarios peruanos: Ninguno.

Observaciones: Hierba conocida de una sola localidad, en la cuenca del Urubamba, donde, al parecer, es especialista de suelos crioturbados, por ello probablemente su rango geográfico sea tan restringido. Este tipo de hábitat es poco conocido botánicamente. No ha vuelto a ser recolectada desde 1982.

\section{Catadysia rosulans O.E. Schulz}

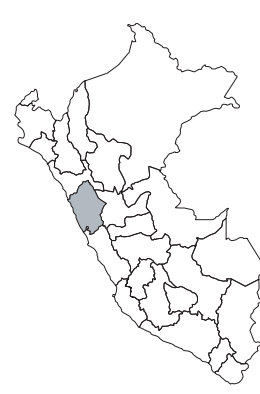

$$
\text { EN, B1a }
$$

Publicación: Notizbl. Bot. Gart. BerlinDahlem 10: 559. 1929.

Colección tipo: A. Weberbauer 7236

Herbarios: B.

Nombre común: Desconocido.

Registro departamental: AN.

Regiones Ecológicas: AA; 4300—4700 $\mathrm{m}$.

SINANPE: PNH

Herbarios peruanos: USM (1).

Observaciones: Hierbas arrosetadas conocidas solamente de unas poblaciones aisladas, en áreas de suelos crioturbados. Esta especie es la única representante de este género endémico. Requiere de estudios de campo para evaluar el estado de sus poblaciones fuera de áreas protegidas.

\section{Dactylocardamum imbricatifolium Al-Shehbaz}

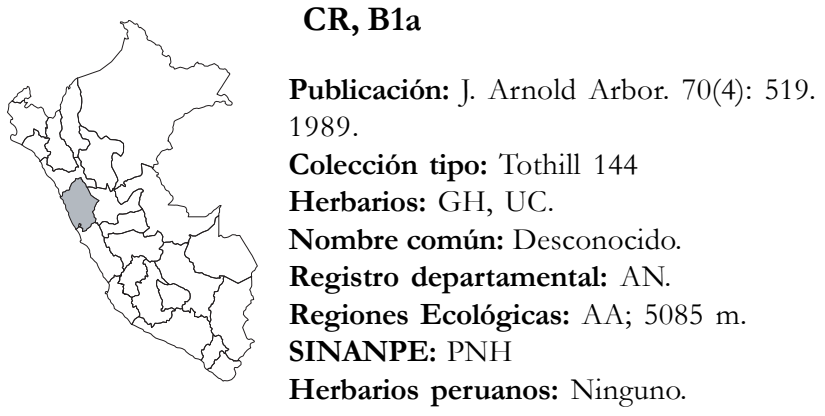

Observaciones: Esta especie herbácea es la única representante del género y se conoce solamente de una localidad, la cual se halla hoy en el Parque Nacional Huascarán. No ha vuelto a ser recolectada desde 1959, tal vez por la escasa herborización de los ambientes altoandinos.

\section{Dictyophragmus englerianus (Musch.) O. E. Schulz}

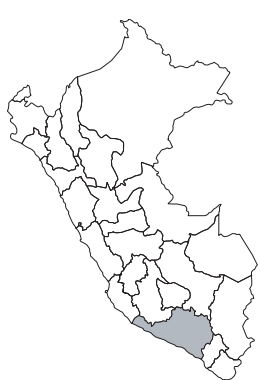
EN, B2c(i,ii)

Publicación: Bot. Jahrb. Syst. 66: 92. 1933. Colección tipo: A. Weberbauer 1453 Herbarios: B, GH, F, K, MO. Nombre común: Desconocido. Registro departamental: AR. Regiones Ecológicas: DST; 100—200 m. SINANPE: Sin registro.

Herbarios peruanos: Ninguno.

Observaciones: Esta especie herbácea no fue considerada en Brako \& Zarucchi (1993) como endémica; sin embargo, las poblaciones conocidas de esta especie se hallan en el sur del país. Fue recolectada originalmente en ambientes de lomas de los alrededores de Mollendo, en 1902. El hábitat de esta especie se halla naturalmente fragmentado. 


\section{Draba argentea O.E. Schultz}

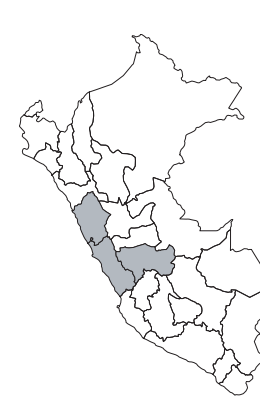

EN, B1a

Publicación: Pflanzenr. IV, 105 (Heft 89): 134. 1927.

Colección tipo: A. Weberbauer 5114; A.W. Hill 13

Herbarios: B.

Nombre común: Desconocido

Registro departamental: AN, JU, LI.

Regiones Ecológicas: PSH, AA; 4250$5800 \mathrm{~m}$.

SINANPE: Sin registro.

Herbarios peruanos: USM (4).

Observaciones: Hierba conocida de cuatro localidades en el centro del país, donde habita ambientes altoandinos, que en general han recibido poca herborización, contribuyendo a lo escaso de su representación en colecciones nacionales. Las amenazas potenciales al hábitat de esta especie incluyen la actividad minera y cambios climáticos.

\section{Draba cuzcoensis O.E. Schultz}

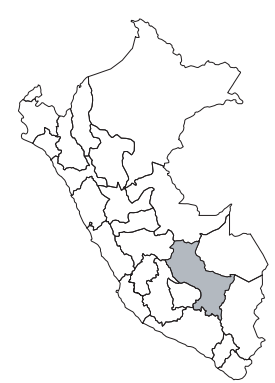

\section{CR, B1a}

Publicación: Bull. Misc. Inform. Kew 1936: 490. 1936.

Colección tipo: D. Stafford 264

Herbarios: K.

Nombre común: Desconocido.

Registro departamental: CU.

Regiones Ecológicas: PSH; $4000 \mathrm{~m}$.

SINANPE: Sin registro.

Herbarios peruanos: Ninguno.

Observaciones: Hierba conocida solamente de una localidad, en la cuenca del Vilcanota, donde no ha vuelto a ser recolectada desde los años 1930. Al igual que otras endémicas en la familia, habita ambientes altoandinos. La localidad original está sujeta a la ganadería y agricultura, por lo que probablemente estas actividades amenacen las poblaciones de esta especie.

\section{Draba matthioloides Gilg \& O.E. Schultz subsp. matthioloides}

\section{EN, B1a}

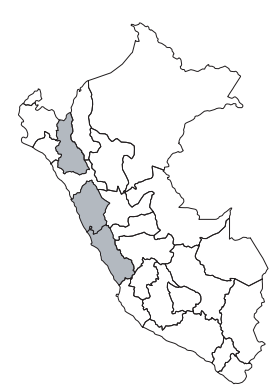

Publicación:

Colección tipo: A. Weberbauer 4231; A. Weberbauer 7019

Herbarios: B; MOL!

Nombre común: Desconocido.

Registro departamental: AN, CA, LI.

Regiones Ecológicas: PSH, AA; 4000$4300 \mathrm{~m}$.

SINANPE: Sin registro.

Herbarios peruanos: MOL (sintipos), USM (1).

Observaciones: Este taxón se conoce de tres localidades en el nor-centro del país. La localidad original se halla en una subcuenca del Marañón. Al parecer, el registro más reciente data de 1952. La minería y pastoreo intensivo podrían considerarse amenazas.

\section{Draba matthioloides Gilg \& O.E. Schultz subsp. saundersii Al-Shehbaz}

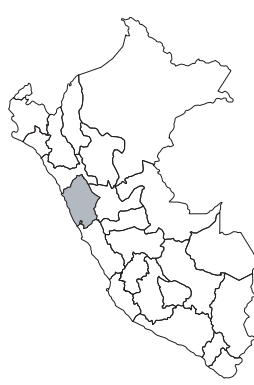

$$
\text { CR, B1a }
$$

Publicación: Novon 1(2): 70. 1991.

Colección tipo: Saunders 926

Herbarios: K.

Nombre común: Desconocido.

Registro departamental: AN.

Regiones Ecológicas: PSH; $4117 \mathrm{~m}$.

SINANPE: Sin registro.

Herbarios peruanos: Ninguno.

Observaciones: Este taxón subarbustivo se conoce solamente de una sola localidad en Ancash y no en La Libertad como se indica en la publicación original. Esta localidad, en los alrededores de Pelagatos, incluye otros endemismos y debería ser de interés a botánicos y conservacionistas. Amenazas a poblaciones de este taxón están asociadas a pastoreo intensivo, incendios intencionales y actividades mineras.

\section{Draba ochropetala O.E. Schultz}

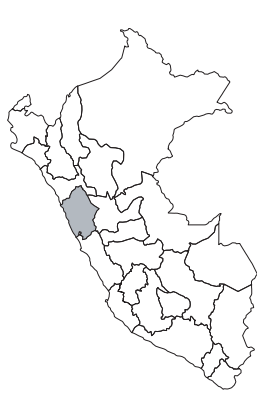

\section{EN, B1ab(iii)}

Publicación: Pflanzenr. IV, 105 (Heft 89): 154-155. 1927.

Colección tipo: A. Weberbauer 7233

Herbarios: B.

Nombre común: Desconocido.

Registro departamental: AN.

Regiones Ecológicas: PSH, AA; 3800$4600 \mathrm{~m}$.

SINANPE: Sin registro.

Herbarios peruanos: USM (2).

Observaciones: Subarbusto conocido de dos localidades, una en la Cordillera Pelagatos y otra en La Negra, en donde las poblaciones están conformadas por individuos aislados o agrupados en números pequeños. Esta especie altoandina puede estar amenazada por la quema asociada a la ganadería.

\section{Draba sagasteguii Al-Shehbaz}

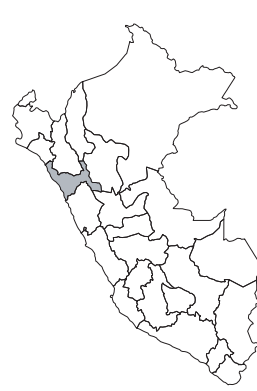

\section{DD}

Publicación: Novon 14(3): 249, 251252, f. 2. 2004.

Colección tipo: A. Sagástegui A. \& M. Zapata 17046

Herbarios: F, MO; $\underline{\mathrm{HAO}}$

Nombre común: Desconocido.

Registro departamental: LL.

Regiones Ecológicas: PAR; $4000 \mathrm{~m}$.

SINANPE: Sin registro.

Herbarios peruanos: HAO (isotipo citado).

Observaciones: Hierba paramuna conocida solamente de la localidad, en los alrededores de una mina, donde fue recolectada en 2002. Se desconoce el estado de las poblaciones de esta especie.

\section{Draba solitaria O.E. Schultz}

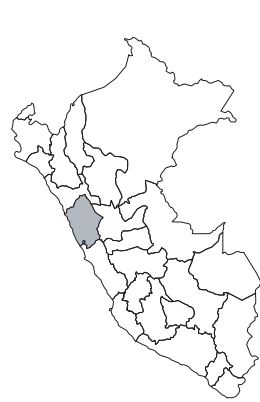

\section{CR, B1a}

Publicación: Pflanzenr. IV, 105 (Heft 89): 155. 1927.

Colección tipo: A. Weberbauer 7235

Herbarios: B, US.

Nombre común: Desconocido.

Registro departamental: AN.

Regiones Ecológicas: PSH; $4450 \mathrm{~m}$.

SINANPE: Sin registro.

Herbarios peruanos: Ninguno. 
Observaciones: Hierba conocida solamente de una localidad, en la Cordillera Pelagatos, la cual alberga otros endemismos y debería ser de interés a botánicos y conservacionistas. $\mathrm{Al}$ parecer, no ha vuelto a ser recolectada desde 1920. Amenazas a sus poblaciones están asociadas a incendios intensionales y pastoreo intensivo.

\section{Draba wurdackii Al-Shehbaz}

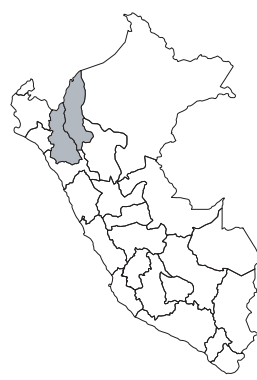

$$
\text { EN, B1a }
$$

Publicación: Novon 1(2): 68-69. 1991. Colección tipo: J.J. Wurdack 1577

Herbarios: US.

Nombre común: Desconocido.

Registro departamental: AM, CA.

Regiones Ecológicas: PAR; 3360—3900 m.

SINANPE: Sin registro.

Herbarios peruanos: CPUN (2).

Observaciones: Esta especie herbácea se conoce de dos localidades paramunas, en el norte del país. Al igual que otros miembros de la familia que habitan la parte altoandina no está bien representada en los herbarios. Amenazas a las poblaciones de esta especie provienen de la actividad minera y expansión agrícola.

\section{Englerocharis pauciflora Al-Shehbaz}

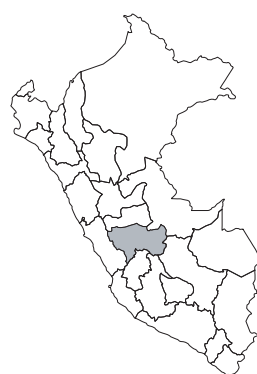

\section{DD}

Publicación: Nordic J. Bot. 8(6): 624. 1989.

Colección tipo: D.N. Smith 5684

Herbarios: GH, MO; USM!.

Nombre común: Desconocido.

Registro departamental: JU.

Regiones Ecológicas: PSH; 3950 m.

SINANPE: Sin registro.

Herbarios peruanos: USM (isotipo).

Observaciones: Especie herbácea conocida solamente de la colección tipo, una planta recolectada en 1983, de la cuenca alta del Comas, donde crecía en pajonal de puna.

\section{Eremodraba schulzii Al-Shehbaz}

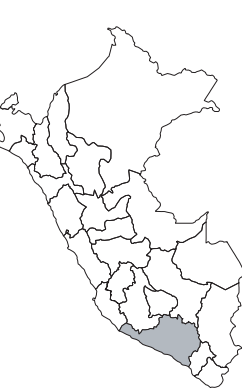

\section{CR, B1a}

Publicación: Ann. Missouri Bot. Gard. 77: 602_604. 1990.

Colección tipo: R. Hirsch P508

Herbarios: B, GH, OXF.

Nombre común: Desconocido.

Registro departamental: AR.

Regiones Ecológicas: MA; 2500-3050 m.

SINANPE: Sin registro.

Herbarios peruanos: Ninguno.

Observaciones: Hierba conocida solamente de dos localidades en el sur del país. Fue descrita de una planta recolectada en la cuenca del Chili; al parecer, no ha vuelto a ser registrada desde 1957. Amenazas a sus poblaciones podrían estar asociadas a la expansión urbana y modificación del hábitat.

\section{Eudema incurva Al-Shehbaz}

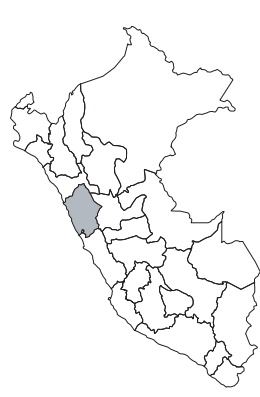

CR, B1a

Publicación: J. Arnold Arbor. 71(1): 101-103, f. 3. 1990

Colección tipo: U. Molau \& B. Eriksen 3512

Herbarios: GH.

Nombre común: Desconocido.

Registro departamental: AN.

Regiones Ecológicas: PSH; 4200— 4400 m.

SINANPE: Sin registro.

Herbarios peruanos: Ninguno.

Observaciones: Hierba terrestre conocida aparentemente sólo de la colección tipo, una planta recolectada en 1987 de las partes altas de la cuenca del Santa. Pastoreo intensivo e incendios asociados podrían ser amenazas a esta especie.

\section{Lepidium cyclocarpum Thell. var. crassius C.L. Hitchc.}

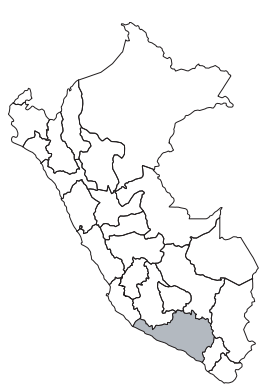

\section{DD}

Publicación: Lilloa 11: 94. 1945.

Colección tipo: C.R. Worth \& J.L. Morrison 15701

Herbarios: C, G, MO, UC.

Nombre común: Desconocido.

Registro departamental: AR.

Regiones Ecológicas: DST; $700 \mathrm{~m}$.

SINANPE: Sin registro.

Herbarios peruanos: Ninguno.

Observaciones: Hierba perenne conocida aparentemente sólo de una localidad, donde fue recolectado en 1938.

\section{Lepidium kalenbornii C.L. Hitchc.}

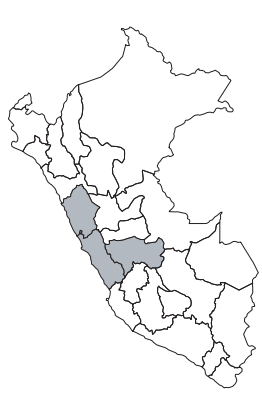

\section{EN, B1a}

Publicación: Lilloa 11: 88-89. 1945.

Colección tipo: A.S. Kalenborn 20

Herbarios: GH, NY, US.

Nombre común: Desconocido.

Registro departamental: AN, JU, LI.

Regiones Ecológicas: PSH, AA; 3048$4600 \mathrm{~m}$.

SINANPE: PNH

Herbarios peruanos: USM (5).

Observaciones: Esta especie herbácea es conocida de varias localidades en las partes altas de la Cordillera occidental. Esta especie fue descrita de una planta recolectada en la cuenca del Mantaro. Se conoce una población en el Parque Nacional Huascarán.

\section{Lepidium pubescens Desv.}

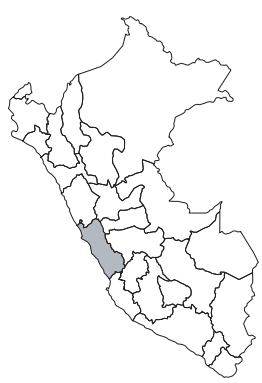

\section{EN, B1a}

Publicación: J. Bot. 3: 165, 180. 1814.

Colección tipo: J. Dombey s.n.

Herbarios: P.

Nombre común: Desconocido.

Registro departamental: LI.

Regiones Ecológicas: MA; 1500-1600 m.

SINANPE: Sin registro.

Herbarios peruanos: Ninguno.

Observaciones: Hierba terrestre conocida del centro del país, de unas pocas localidades en la cuenca del Rímac. Ha sido registrada en el siglo XVIII y luego en 1910 y 1930. Amenazas a sus poblaciones están asociadas al deterioro ambiental de esa cuenca. 


\section{Mathewsia matthioloides (Schlecht.) C. Muell.}

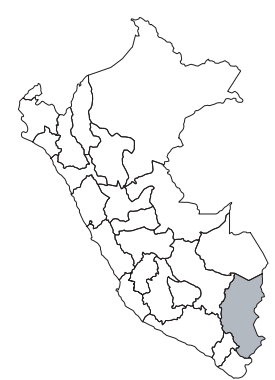

\section{DD}

Publicación: Ann. Bot. Syst. 7: 144. 1868 Colección tipo: W. Lechler 1702

Herbarios: G.

Nombre común: Desconocido.

Registro departamental: PU.

Regiones Ecológicas: Sin datos; altitud desconocida.

SINANPE: Sin registro.

Herbarios peruanos: Ninguno.

Observaciones: Hierba terrestre conocida solamente de la colección tipo, una planta recolectada en el siglo XIX en el sur del país. Poco se sabe de las condiciones de sus poblaciones.

\section{Mathewsia peruviana O.E. Schulz}

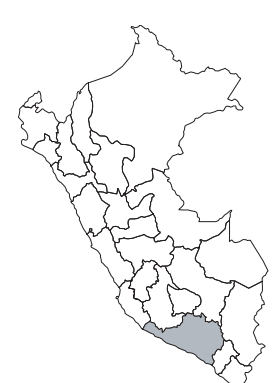

EN, B2c(i,ii)

Publicación: Notizbl. Bot. Gart. BerlinDahlem 10: 562. 1929.

Colección tipo: A. Raimondi 11632

Herbarios: B; USM!.

Nombre común: Desconocido.

Registro departamental: AR.

Regiones Ecológicas: DST; 100—700 m. SINANPE: Sin registro.

Herbarios peruanos: USM (isotipo+5).

Observaciones: Esta especie herbácea se conoce de ambientes rocosos, en dos localidades de lomas, en el sur del Perú. Estas lomas no reciben protección por el estado y están afectas a la contaminación ambiental y deterioro por pastoreo intensivo. Las colecciones más recientes fueron realizadas aparentemente en 1957.

\section{Sisymbrium arequipanum Al-Shehbaz}

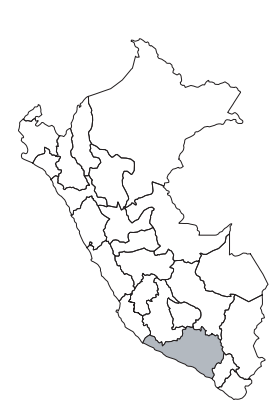

\section{EN, B1a}

Publicación: Ann. Missouri Bot. Gard. 76(4): 1176-1177, f. 1. 1989.

Colección tipo: D. Stafford 674

Herbarios: F.

Nombre común: Desconocido.

Registro departamental: AR.

Regiones Ecológicas: PSH, AA; 3950$4423 \mathrm{~m}$.

SINANPE: Sin registro.

Herbarios peruanos: Ninguno.

Observaciones: Esta hierba es conocida, al parecer, de dos poblaciones naturalmente fragmentadas en el sur del país. Los ejemplares conocidos provienen de laderas con suelos deleznables, de dos volcanes cerca de la ciudad de Arequipa. La colección tipo fue recolectada en 1937.

\section{Sisymbrium effusum O.E. Schulz}

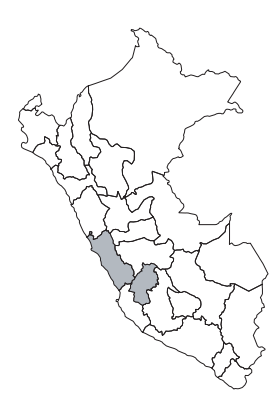

\section{EN, B1a}

Publicación: Pflanzenr. IV, 105 (Heft 86): 72-73. 1924.

Colección tipo: A. Weberbauer 6499

Herbarios: B, US; MOL!, USM!.

Nombre común: Desconocido.

Registro departamental: HV, LI.

Regiones Ecológicas: MA; 2800—3350 m.

SINANPE: RPNYC

Herbarios peruanos: MOL (isotipo), USM

(isotipo+4).
Observaciones: Especie arbustiva, poco frecuente, conocida de dos localidades en el centro del país. Ambas localidades están ubicadas en áreas poco herborizadas. Las poblaciones de esta especie son pequeñas y dispersas.

\section{Sisymbrium ferreyrae Forther $\&$ Weigend}

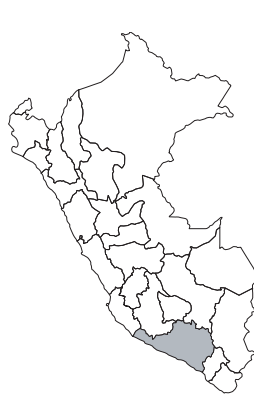

\section{CR, B1a}

Publicación: Brittonia 51(2): 120. 1999.

Colección tipo: M. Weigend \& H. Forther 97/739

Herbarios: F, MSB, NY; USM!.

Nombre común: Desconocido.

Registro departamental: AR.

Regiones Ecológicas: DST; 350—960 m.

SINANPE: Sin registro.

Herbarios peruanos: USM (isotipo).

Observaciones: Esta especie herbácea fue descrita de una planta recolectada en 1997 de ambientes de lomas. El hábitat es uno de los más propensos a ser modificados y destruidos por la actividad humana; por ello se aplica la categoría Crítica.

\section{Sisymbrium lactucoides Forther \& Weigend}

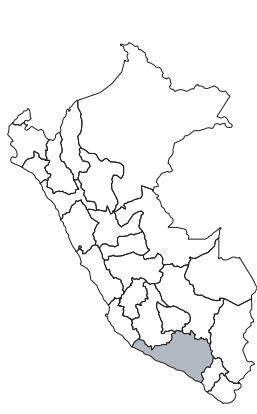

\section{CR, B1a}

Publicación: Brittonia 51(2): 120, f. 2. 1999.

Colección tipo: M. Weigend \& H. Forther $97 / 740$

Herbarios: F, MSB, NY; HUT!, USM!.

Nombre común: Desconocido.

Registro departamental: AR.

Regiones Ecológicas: DST; 350—960 m.

SINANPE: Sin registro.

Herbarios peruanos: HUT (isotipo), USM (isotipo).

Observaciones: Esta especie herbácea, al igual que otra endémica, Sisymbrium ferreyrae, fue descrita de una colección reciente. El hábitat de la que se conoce es uno de los más propensos a ser modificados y destruidos por la actividad humana; por ello se aplica la categoría Crítica.

\section{Sisymbrium llatasii Al-Shehbaz}

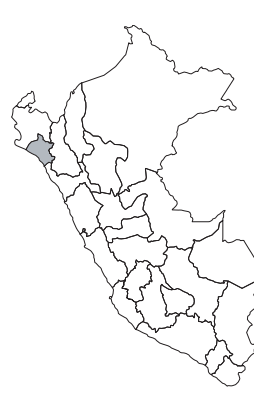

\section{DD}

Publicación: Ann. Missouri Bot. Gard. 77(1): 219, f. 1. 1990.

Colección tipo: S. Llatas Q. 2102

Herbarios: F, GH.

Nombre común: Desconocido.

Registro departamental: LA.

Regiones Ecológicas: DCT; $580 \mathrm{~m}$.

SINANPE: Sin registro.

Herbarios peruanos: HAO (1).

Observaciones: Esta especie anual se conoce de una localidad, en el norte del país, en lo que se considera la expresión más septentrional del ambiente de lomas. No se conoce el estatus de conservación de sus poblaciones. 


\section{Sisymbrium morrisonii Al-Shehbaz}

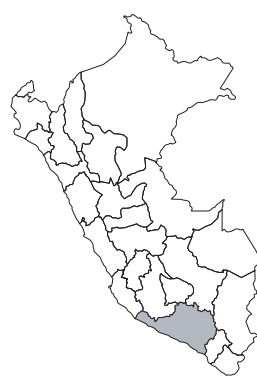

Publicación: Ann. Missouri Bot. Gard. 77(1): 219, f. 2. 1990.

Colección tipo: C.R. Worth \& J.L. Morrison 15729

Herbarios: UC.

Nombre común: Desconocido.

Registro departamental: AR.

Regiones Ecológicas: DST; $700 \mathrm{~m}$.

SINANPE: Sin registro.

Herbarios peruanos: Ninguno.

Observaciones: Hierba descrita de una planta recolectada en 1938, de los alrederores de Mollendo. Este taxón fue considerado por Brako \& Zarucchi (1993) como un endemismo; sin embargo, no ha sido posible evaluarlo, ni asignarle una categoría.

\section{Sisymbrium pectinifolium Al-Shehbaz}

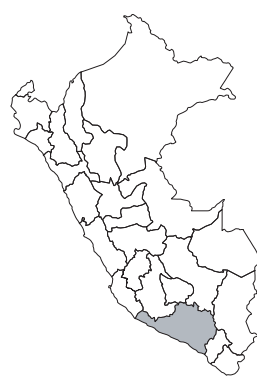

Publicación: Harvard Pap. Bot. 2: 14, f. 2. 1990 .

Colección tipo: R.M. Straw 2380

Herbarios: US.

Nombre común: Desconocido.

Registro departamental: AR.

Regiones Ecológicas: MA; $2820 \mathrm{~m}$.

SINANPE: Sin registro.

Herbarios peruanos: Ninguno.

Observaciones: Este taxón fue considerado por Brako \& Zarucchi (1993) como un endemismo; sin embargo, no ha sido posible evaluarlo, ni asignarle una categoría.

\section{Sisymbrium weberbaueri O.E. Schulz}

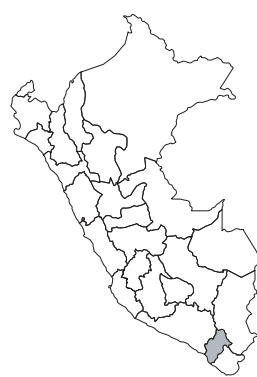

Publicación: Publ. Field Columbian Mus., Bot. Ser. 8(2): 80. 1930.

Colección tipo: A. Weberbauer 7426

Herbarios: F.

Nombre común: Desconocido.

Registro departamental: MO.

Regiones Ecológicas: MDE; 1800—2000 m.

SINANPE: Sin registro.

Herbarios peruanos: Ninguno.

Observaciones: Este taxón fue considerado por Brako \& Zarucchi (1993) como un endemismo; sin embargo, no ha sido posible evaluarlo, ni asignarle una categoría.

\section{Weberbauera bracteata (O.E. Schulz) J.F. Macbr.}

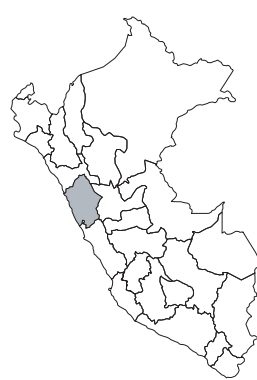

\section{CR, B1a}

Publicación: Candollea 5: 356. 1934. Colección tipo: A. Weberbauer 7234 Herbarios: B, F.

Nombre común: Desconocido.

Registro departamental: AN.

Regiones Ecológicas: AA; $4600 \mathrm{~m}$.

SINANPE: Sin registro.

Herbarios peruanos: Ninguno.

Observaciones: Hierba altoandina conocida solamente de la colección tipo, una planta recolectada en 1920, en la Cordillera Pelagatos, localidad que hospeda varios endemismos y debería ser de interés a botánicos y conservacionistas. Amenazas a sus poblaciones podrían estar asociadas a actividades de pastoreo.

\section{Weberbauera dillonii Al-Shehbaz}

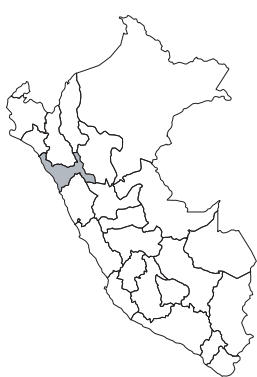

CR, B1a

Publicación: Novon 14(3): 261, f. 1. 2004. Colección tipo: I. Sánchez V. et al. 11172 Herbarios: F, MO; CPUN!.

Nombre común: Desconocido.

Registro departamental: LL.

Regiones Ecológicas: PAR; $3000 \mathrm{~m}$.

SINANPE: Sin registro.

Herbarios peruanos: CPUN (isotipo).

Observaciones: Hierba paramuna conocida de una sola localidad en el noreste de La Libertad. Esta especie fue descrita de una planta recolectada en 2001, en la cuenca del Marañón, en los alrededores de un nevado hoy desaparecido.

\section{Weberbauera perforata Al-Shehbaz}

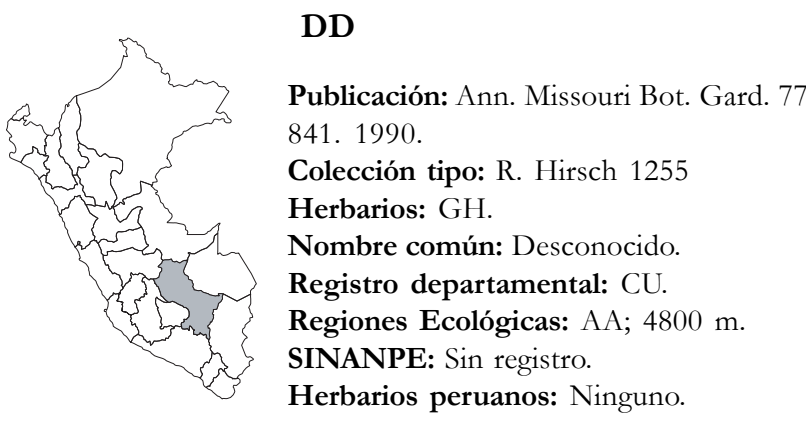

Observaciones: Hierba arrosetada, conocida solamente de la colección tipo, una planta recolectada en 1957 de los alrededores de un nevado en el sureste del Departamento de Cusco. Esta zona ha sido escasamente herborizada.

\section{Weberbauera scabrifolia Al-Shehbaz}

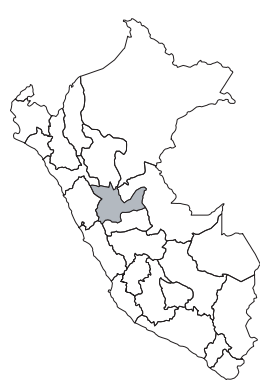

\section{NE}

Publicación: Novon 14(3): 264-266, f. 2. 2004.

Colección tipo: M. Weigend et al. 5520

Herbarios: M, MO; HUT!

Nombre común: Desconocido.

Registro departamental: HU.

Regiones Ecológicas: PSH; $4030 \mathrm{~m}$.

SINANPE: Sin registro.

Herbarios peruanos: HUT (isotipo).

Observaciones: Hierba conocida aparentemente sólo de la colección tipo, una planta recolectada en 2001, en la cuenca alta del Marañón. Esta parte de la cuenca ha sido escasamente herborizada. Esta especie fue recolectada en ambientes de suelos someros sobre rocas cálcicas.

\section{Weberbauera smithii Al-Shehbaz}

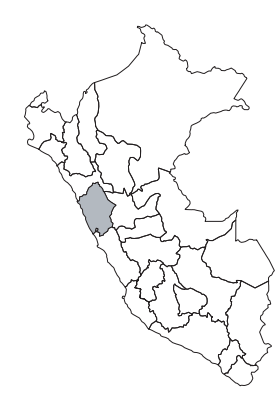

Publicación: J. Arnold Arbor. 71(2): 233-234, f. 4. 1990.

Colección tipo: D.N. Smith \& K. Goodwin 8894

Herbarios: MO; USM!.

Nombre común: Desconocido.

Registro departamental: AN.

Regiones Ecológicas: AA; $4770 \_4870 \mathrm{~m}$.

SINANPE: PNH

Herbarios peruanos: USM (isotipo+1). 
Observaciones: Hierba conocida solamente de una localidad, ubicada en el Parque Nacional Huascarán. Al igual que otras especies altoandinas, las poblaciones son escasas y poco herborizadas. Probablemente se hallen otros individuos fuera de esta localidad y si fuera así la actividad minera y el pastoreo podrían ser amenazas potenciales.

\section{Weberbauera violacea Al-Shehbaz}

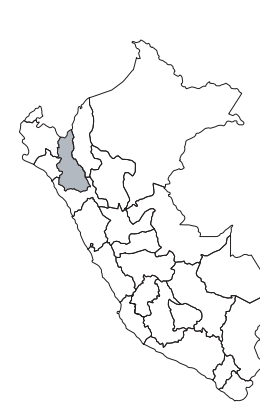

DD

Publicación: Novon 14(3): 266-268, f. 3. 2004.

Colección tipo: A. Sagástegui A. et al. 11175

Herbarios: MO; HUT!

Nombre común: Desconocido.

Registro departamental: CA.

Regiones Ecológicas: PAR; $3800 \mathrm{~m}$.

SINANPE: Sin registro.

Herbarios peruanos: HUT (isotipo).

Observaciones: Esta hierba paramuna es conocida de la localidad tipo, en la cuenca del Crisnejas, un tributario del Marañón. El ejemplar tipo fue recolectado en 1983 , de una jalca poco herborizada. 